\title{
Modulatory Role of Single Nucleotide Polymorphisms of Distinct Genetic Pathways on Clinical Behavior of Medullary Thyroid Carcinoma
}

\author{
Vasudha Mishra $^{1,2}$, Pradnya Kowtal ${ }^{1,2}$, Pallavi Rane ${ }^{3}$, Rajiv Sarin ${ }^{1,2,4 *}$
}

\begin{abstract}
Background: Role of RET proto-oncogene as predisposing gene for Medullary Thyroid Carcinoma is well established which provides the basis for clinical management of patients. However clinical behavior of MTC varies considerably among patients. Several studies have investigated whether SNPs in low penetrance genes could modulate the clinical behavior of MTC but with conflicting or inconclusive results. The present study aimed to investigate the modifier effect of 13 SNPs of three distinct genetic pathways -Detoxification, Cell cycle regulation and RET on the clinico-pathological features of hereditary and sporadic MTC. Methods: SNPs were genotyped using RFLP or TaqMan method. The genotypes were correlated with various clinico-pathological parameters (age and calcitonin levels at MTC diagnosis, tumor volume, nodal and distant metastasis). Results: Nodal metastasis was the only clinico-pathological parameter showing significant association with any SNP. In the hereditary MTC group $(n=77)$, incidence of nodal metastases was significantly higher in wild type allele for Cyp1A1m1,CDKN2A and $C D K N 2 C$ ( $\mathrm{p}=0.01$ for all three). In sporadic MTC group $(\mathrm{n}=361) C D K N 2 C$ wild type allele had higher nodal metastasis $(\mathrm{p}=0.03)$. Conclusion: In this largest MTC cohort with comprehensive analysis of modulatory role of 13 most frequently studied SNPs with MTC clinical outcome, we observed a statistically significant association of few SNPs with nodal metastasis. However as these SNPs did not show association with any other clinico-pathological parameters like tumor volume or Calcitonin, they may not be true modifier of MTC. Additional large cohort studies with clinico-pathological details and long-term follow-up are needed to identify genetic modifiers of MTC behavior.
\end{abstract}

Keywords: Medullary Thyroid Carcinoma- MTC- RET- SNP- association-study

Asian Pac J Cancer Prev, 21 (5), 1289-1293

\section{Introduction}

Gain-of-function germline point mutations in the RET proto-oncogene have long been established as the predisposing factor for hereditary Medullary Thyroid Carcinoma (MTC) which presents as a part of an autosomal dominant inherited cancer syndrome MEN2 (De Groot et al., 2006; Donis-Keller et al., 1993; Eng et al., 1996; Figlioli et al., 2013). The site specific mutations in the 'hotspot' regions of RET gene govern phenotypic variability observed in the mutation carriers provide the basis for clinical management of proband along with prophylactic interventions in healthy carriers (Kloos et al., 2009; Wells et al., 2015). Despite the strong genotype-phenotype association, the clinical behavior of MTC varies considerably among patients and clinical heterogeneity is observed in individuals harboring the same RET mutations (Barbieri et al., 2013). Similarly for sporadic MTC, somatic mutation in RET gene is not found in all cases (Agrawal et al., 2013; Marsh et al., 1996) and appear not to occur uniformly among the different subpopulation of cells in the tumor unlike germline mutations (Eng et al., 1996).

Several studies have investigated whether the presence of variants such as Single Nucleotide Polymorphisms (SNPs) in low penetrance genes could modulate the clinical behavior of the disease. Low penetrance gene variants are the polymorphic sequence variants which are known to have a relatively moderate effect in disease progression compared to pathogenic variants. If the presence of an SNP correlates with a change in clinical presentation of the disease then they may be considered as disease modifiers (Robledo et al., 2003). However, it is still a matter of debate to what extent these neutral

${ }^{1}$ Sarin Lab, Advanced Centre for Treatment Research and Education in Cancer, Tata Memorial Centre, Kharghar, Navi Mumbai, India. ${ }^{2}$ Homi Bhabha National Institute, Training School Complex, Anushakti Nagar, Mumbai, 400085, India. ${ }^{3}$ Clinical Research Centre (CRC), Advanced Centre for Treatment Research and Education in Cancer, Tata Memorial Centre, Kharghar, Navi Mumbai. ${ }^{4}$ Cancer Genetics Clinic, Tata Memorial Hospital, Tata Memorial Centre, Parel, Mumbai,400012, India. *For Correspondence: rsarin@actrec.gov.in 
polymorphisms could have modifying roles in the pathogenesis of MEN2 or sporadic MEN2-related tumors.

A series of studies in different population have attempted to correlate presence of SNPs in RET gene with MTC progression but the findings have been contradictory or inconclusive possibly because of small cohort sizes of this relatively rare cancer or due to differences in geo-ethnic background (Fugazzola et al., 2008; Lesueur et al., 2006; Machens et al., 2012; Robledo et al., 2003; Siqueira et al., 2010; Sromek et al., 2010; Tamanaha et al., 2009). Recently SNPs in detoxification and Cell cycle regulatory genes have also been studied as modulators of both hereditary and sporadic MTC behavior (Barbieri et al., 2013, 2014, 2012; Pasquali et al., 2011). However none of the studies have examined the association of SNPs of all these distinct genetic pathways together in a single cohort of MTC cases. Using the largest cohort of 438 MTC cases (361 sporadic and 77 hereditary) we therefore undertook a comprehensive gene dose-response relationship analysis between 13 SNPs of genes of three distinct genetic pathways (Table 1). These include SNPs from genes of detoxification (Cyp1A1m1, Cyp $1 A 2 * F$, $N A T 2, G S T P 1)$, cell cycle regulation (CDKN1A, CDKN1B, $C D K N 2 A, C D K N 2 B, C D K N 2 C$ ) and the RET gene (G691S, L769L,S836S, S904S). This gene dose-response relationship aimed to investigate the clinic-pathological differences between the wild-type versus heterozygous versus homozygous variant for each SNP separately in hereditary and sporadic MTC cases.

\section{Materials and Methods}

\section{Patient cohort}

The 438 Indian MTC patients enrolled in this study were registered at the Cancer Genetics Clinic, Tata Memorial Hospital over a period of 12 years (2006 to 2018), under an Institutional Ethics Committee approved study. For each patient, personal and family history with clinico-pathological details was recorded using a standard case-record form. Blood sample was collected after a written informed consent and detailed genetic counseling. Based on clinical details, family history and RET genetic test result, the patients were classified as hereditary MTC cases or sporadic. In our cohort of 438 MTC patients, we have 77 hereditary and 361 sporadic MTC patients. All the 77 hereditary MTC cases harbored mutations in the hotspot region of the RET gene.

\section{Clinical and Pathological examination}

The clinical diagnosis of MTC was made on biochemical evaluation of serum Calcitonin levels at diagnosis (reference range: $2-10 \mathrm{mg} / \mathrm{ml}$ ) and histopathological examination of surgically resected tumor tissues. The tumor volume was calculated using the ellipsoid volume formula $1 / 2 \times 1 \times \mathrm{w} \times \mathrm{h} \mathrm{cm}^{3}$ (Jensen et al, 2008). The diagnosis of nodal metastasis was made on the histopathological examination of lymph nodes resected at the time of total thyroidectomy. Distant metastasis was confirmed by either ultrasonography, computed tomography, magnetic resonance imaging, positron emission tomography or their combination. The age at
MTC diagnosis was recorded for each patient.

\section{Genotyping}

DNA was extracted from peripheral blood sample using Qiagen QIAmp DNA Mini kit (Cat\#51304). Germline RET mutation analysis was performed for 6 hotspot exons of RET $(10,11,13,14,1516)$ by PCR and Sanger Sequencing. Sanger Sequencing was performed using BigDye Terminator Cycle Sequencing kit v3.1 (Applied Biosystems) on ABI 3500 and 3730 DNA Sequencer (Applied Biosystems) and electropherograms were analyzed by Chromas Lite version 2.6.4 using reference sequence of RET gene from National Centre for Biotechnology Information (NCBI) NG_007489.1.

SNP genotyping was performed by Restriction Fragment Length Polymorphism (RFLP) for Cyp $1 A 1 \mathrm{~m} 1$, Cyp1A2*F, GSTP1, NAT2, CDKN1A, CDKN1B, CDKN2A, RET L769L, S836S and $S 904 S$ polymorphisms and by TaqMan SNP genotyping method for $C D K N 2 B$, CDKN2C and RET G691S polymorphisms. For RFLP, the digested products were visualized on $2 \%$ agarose gel and the genotypes were inferred from band sizes in the gel. For TaqMan SNP genotyping, realtime PCR was performed on QuantStudio 5.0 and genotypes were inferred from amplification plot and allelic discrimination plots. Around $5 \%$ of all the genotyping results were confirmed on Sanger Sequencing.

\section{Statistical Analysis}

All Statistical analysis was performed on SPSS v21.0. The clinico-pathological variables used for correlation studies included age at MTC diagnosis, serum Calcitonin levels at diagnosis (Pre-Op), tumor volume $\left(\mathrm{cm}^{3}\right)$, nodal and distant metastasis. To examine gene dose-response relationship, the clinic-pathological variables were stratified between wild-type, heterozygous and homozygous variants and compared. Categorical data was tested with Pearson Chi-Square test and two-tailed Fisher exact test whereas for continuous data, the means were compared using one-way analysis of variance (ANOVA) and medians was compared using Kruskal-Wallis test. The level of significance was set at $<0.05$.

\section{Results}

The 438 Indian MTC cases in our cohort included 239 males and 199 females. The mean age at MTC diagnosis was $40.64+14.24$; median: 40 years (range: 8 -80years). All the SNPs except Cyp1A1m1 and CDKN2A maintained the Hardy-Weinberg equilibrium (Table 2). The genotype frequency of the wild-type, heterozygous and homozygous variants for all the 13 SNPs are given in Table 3. For a few SNPs, the genotype frequency of the homozygous variant is either absent or very low limiting their analysis between the wildtype and heterozygous variants.

In gene dose-response association, the only clinic-pathological parameter with which any SNP showed significant association was nodal metastasis at diagnosis. In hereditary MTC group, Cyp $1 A 1 \mathrm{~m} 1, C D K N 2 A$ and CDKN2C SNP showed this association (Supplementary 
Table 1. List of SNPs of Distinct Genetic Pathways Selected for the Study

\begin{tabular}{|c|c|c|c|c|c|}
\hline Gene/SNP & Reference ID & Variant & Nucleotide change & Ancestral Allele & Amino acid Change \\
\hline \multicolumn{6}{|c|}{ Genes of Detoxification } \\
\hline Cyp1A1m1 & rs4646903 & 3'UTR & $\mathrm{T} / \mathrm{C}$ & $\mathrm{T}$ & - \\
\hline Cyp1A2 & rs 762551 & Intronic & $\mathrm{A} / \mathrm{C}$ & A & - \\
\hline NAT2 & rs 1041983 & Synonymous & $\mathrm{C} / \mathrm{T}$ & $\mathrm{C}$ & Y94Y \\
\hline GSTP1 & rs 1695 & Missense & $\mathrm{A} / \mathrm{G}$ & A & $\mathrm{I} 105 \mathrm{~V}$ \\
\hline \multicolumn{6}{|c|}{ Cell Cycle regulatory genes } \\
\hline CDKN1A & rs 1801270 & Missense & $\mathrm{C} / \mathrm{A}$ & $\mathrm{C}$ & S31R \\
\hline$C D K N 1 B$ & rs2066827 & Missense & $\mathrm{T} / \mathrm{G}$ & $\mathrm{T}$ & V109G \\
\hline$C D K N 2 A$ & rs11515 & 3' UTR & $\mathrm{G} / \mathrm{C}$ & $\mathrm{G}$ & - \\
\hline$C D K N 2 B$ & rs 1063192 & 3'UTR & $\mathrm{T} / \mathrm{C}$ & $\mathrm{T}$ & - \\
\hline CDKN2C & rs12885 & 3' UTR & $\mathrm{G} / \mathrm{T}$ & G & - \\
\hline \multicolumn{6}{|c|}{ RET Proto oncogene } \\
\hline G691S RET & rs1799939 & Missense & $\mathrm{G} / \mathrm{A}$ & G & G691S \\
\hline L769L RET & rs 1800861 & Synonymous & $\mathrm{T} / \mathrm{G}$ & $\mathrm{T}$ & L769L \\
\hline S836S RET & rs 1800862 & Synonymous & $\mathrm{C} / \mathrm{T}$ & $\mathrm{C}$ & S836S \\
\hline S904S RET & rs 1800863 & Synonymous & $\mathrm{C} / \mathrm{G}$ & $\mathrm{C}$ & S904S \\
\hline
\end{tabular}

Table S1). Patients with wildtype Cyp $1 A 1 \mathrm{~m} 1$ showed higher rate of regional nodal metastasis compared to the heterozygous and homozygous variants $(95.6 \%$ vs $86.2 \%$ vs $33.3 \%$; $=0.01)$. Similarly patients with wildtype $C D K N 2 A$ showed a higher incidence of nodal metastasis compared to their variant counterparts $(91.3 \%$ vs $83.3 \%$ vs $33.3 \%$; $\mathrm{p}=0.01)$. For $C D K N 2 C$, the comparison could only be made between wildtype and heterozygote as the homozygous variant for this SNP was absent. We observed that cases heterozygous for CDKN2C SNP showed higher rate of lymph node metastasis compared to wildtype CDKN2C (100\% vs 86.3\%; p=0.01) (Supplementary Table S1).

In sporadic MTC group, this association was observed between the wildtype and the homozygous variant for $C D K N 2 C S N P$. The cases wildtype for CDKN2C SNP showed higher rate of nodal metastasis

Table 2. Hardy-Weinberg (HWE) Calculation for SNPs in MTC Cohort

\begin{tabular}{lcc}
\hline Gene/SNP & \multicolumn{2}{c}{ MTC cases $(\mathrm{n}=438)$} \\
& MAF & HWE $P$-value \\
\hline Cyp1A1m1 & 0.31 & 0.006 \\
Cyp1A2 & 0.43 & 0.65 \\
NAT2 & 0.45 & 1.00 \\
GSTP1 & 0.26 & 0.65 \\
CDKN1A & 0.09 & 0.14 \\
CDKN1B & 0.29 & 0.57 \\
CDKN2A & 0.07 & 0.003 \\
CDKN2B & 0.25 & 0.11 \\
CDKN2C & 0.10 & 0.31 \\
G691S RET & 0.27 & 0.17 \\
L769L RET & 0.35 & 0.60 \\
S836S RET & 0.08 & 0.33 \\
S904S RET & 0.29 & 0.05 \\
\hline
\end{tabular}

compared to homozygous variant genotype $(83.8 \%$ vs $40 \% ; p=0.03)$. The rate of nodal metastasis for heterozygous variant was similar to the wildtype $C D K N 2 C$ (84.4\% vs $83.8 \%$ ) (Supplementary Table S2). No other significant association was observed between any of the SNPs and the patient's clinico-pathological behavior.

\section{Discussion}

The role of RET variants as modulators of clinical behavior of MTC has been studied in different populations in both hereditary and sporadic MTC patients. A few studies have reported a significant dose-response relationship between the RET SNPs and the age at MTC diagnosis (Cardot-Bauters et al., 2008; Robledo et al., 2003; Siqueira et al., 2010; Sromek et al., 2010) whereas many others have failed to establish these associations(Fugazzola et al., 2008; Lesueur et al., 2006; Machens et al., 2012; Tamanaha et al., 2009). Recently, Barbieri et al., (2012, 2013) have studied the modulatory role of SNPs of genes of detoxification (Cyp1A1m1, Cyp1A2*F, NAT2 and GSTP1) in both hereditary and sporadic MTC patients in Brazilian population (Barbieri et al., 2012, 2013). In their study on sporadic MTC patients, they failed to establish any significant relationship between the genetic profiles of these SNPs and patient's clinical outcome which they concluded to be a result of their small cohort size (47 sporadic MTC cases) (Barbieri et al., 2012). Further in their study on hereditary MTC cohort of 132 patients, they demonstrated that the inherited profile of $C y p 1 A 2^{*} F, N A T 2$ and GSTP1 contributed significantly to the clinical behavior of the patients (Barbieri et al., 2013). They showed that inheritance of Cyp $1 A 2 * F S N P$ was associated with lesser tumor burden in patients and inheritance of GSTP1 SNP was associated with later age at MTC diagnosis. The same group also studied the influence of cell cycle regulation genes (CDKN1A, CDKN1B, $C D K N 2 A, C D K N 2 B$ and $C D K N 2 C$ ) in a small cohort of 
Table 3. Genotype Frequency of SNPs in Hereditary and Sporadic MTC Groups

\begin{tabular}{lcccccc}
\hline Gene/ SNP & \multicolumn{2}{c}{ Genotype Frequency - Hereditary MTC cases $(\mathrm{n}=77)$} & \multicolumn{4}{c}{ Genotype Frequency - Sporadic MTC cases (n=361) } \\
& Wild-type & Heterozygous & Homozygous & Wild-type & Heterozygous & Homozygous \\
\hline Cyp1A1m1 & $37(48.1 \%)$ & $36(46.7 \%)$ & $4(5.2 \%)$ & $161(44.6 \%)$ & $175(48.4 \%)$ & $25(6.9 \%)$ \\
Cyp1A2 & $28(36.4 \%)$ & $34(44.2 \%)$ & $15(19.5 \%)$ & $117(32.4 \%)$ & $176(48.7 \%)$ & $68(18.8 \%)$ \\
NAT2 & $31(42.2 \%)$ & $34(44.2 \%)$ & $12(15.6 \%)$ & $103(28.5 \%)$ & $183(50.7 \%)$ & $75(20.7 \%)$ \\
GSTP1 & $35(45.5 \%)$ & $35(45.5 \%)$ & $7(9.0 \%)$ & $208(57.6 \%)$ & $129(35.7 \%)$ & $24(6.6 \%)$ \\
CDKN1A & $65(84.4 \%)$ & $11(14.3 \%)$ & $1(1.3 \%)$ & $295(81.7 \%)$ & $66(18.3 \%)$ & $0(0 \%)$ \\
CDKN1B & $35(45.5 \%)$ & $33(42.8 \%)$ & $9(11.6 \%)$ & $182(50.4 \%)$ & $153(42.4 \%)$ & $26(7.2 \%)$ \\
CDKN2A & $67(87 \%)$ & $7(9.0 \%)$ & $3(3.8 \%)$ & $310(85.8 \%)$ & $47(13.0 \%)$ & $4(1.1 \%)$ \\
CDKN2B & $46(59.8 \%)$ & $27(35.0 \%)$ & $4(5.2 \%)$ & $206(57.1 \%)$ & $125(34.6 \%)$ & $30(8.3 \%)$ \\
CDKN2C & $62(80.5 \%)$ & $14(18.1 \%)$ & $1(1.3 \%)$ & $297(82.3 \%)$ & $59(16.3 \%)$ & $5(1.4 \%)$ \\
G691S RET & $37(48.1 \%)$ & $31(40.2 \%)$ & $9(11.6 \%)$ & $201(55.6 \%)$ & $131(36.3 \%)$ & $29(8.0 \%)$ \\
L769L RET & $35(45.5 \%)$ & $33(42.8 \%)$ & $9(11.6 \%)$ & $146(40.4 \%)$ & $172(47.6 \%)$ & $43(11.9 \%)$ \\
S836S RET & $64(83.1 \%)$ & $13(16.8 \%)$ & $0(0.0 \%)$ & $311(86.1 \%)$ & $46(12.7 \%)$ & $4(1.1 \%)$ \\
S904S RET & $36(46.8 \%)$ & $30(38.9 \%)$ & $11(14.3 \%)$ & $194(53.7 \%)$ & $133(36.8 \%)$ & $34(9.4 \%)$ \\
\hline
\end{tabular}

45 sporadic MTC cases (Barbieri et al., 2014). They have reported that patients with wildtype CDKN1A presented extrathyroidal tumor extension more frequently $(\mathrm{p}=0.037)$ and patients with wildtype $C D K N 2 C$ presented larger tumors $(\mathrm{p}=0.032)$ whereas patients with polymorphic $C D K N 2 B$ presented higher rate of distant metastasis $(\mathrm{p}=0.026)$. Further, one study on Italian population by Pasquali et al., (2011) reported CDKN1B V109G polymorphism to be a prognostic marker in sporadic MTC (Pasquali et al., 2011). The study was performed in a cohort of 84 sporadic MTC patients and 90 matched controls. They had demonstrated that CDKN1B V109G polymorphism was associated with a more favorable disease progression than the wildtype allele.

Although statistically significant associations were observed in some of these studies, their cohort size was comparatively small. A small cohort study is likely to produce false-positive results than the conclusions drawn from a larger cohort study. The two comparatively larger studies investigating the role of these SNPs was by Barbieri et al., (2013) in a cohort of 132 hereditary MTC patients (discussed above) and the other by Machens et al., (2012). Machens et al., (2012) have investigated the clinical relevance of SNPS of RET gene (G691S, L769L, $S 836 S$ and $S 904 S$ ) in a cohort of 150 sporadic MTC cases. They investigated the differences in clinic-pathological characteristics of sporadic MTC patients with or without these RET SNPS. They have failed to identify any significant dose-response relationship between the RET SNPS and MTC clinical behavior. The findings of the two studies had called for another comprehensive and larger cohort study to decipher definitive conclusions.

The present study represents a large cohort of MTC including both the hereditary and sporadic MTC cases. Here we have undertaken a comprehensive investigation of 13 SNPs of all the above discussed genetic pathways in a cohort of 361 sporadic and 77 hereditary MTC cases. Although a few SNPs show a statistically significant association $(\mathrm{p}<0.01)$ with regional nodal metastasis, they may not represent a true gene dose-response relationship as they were not associated with any other clinico-pathological parameters. Our findings on RET SNPS were consistent with Machens et al., (2012) group as we too have failed to establish any gene dose-response relationship between the carriers and non-carriers of RET gene SNPs in our large MTC cohort. Further, our study have failed to replicate the findings of Pasquali et al group as we have not identified any significant association between the CDKNIB SNP and the patients clinical outcome. This discrepancy might have arisen as we did not have the follow-up data of the patients with which Pasquali et al have done their correlation studies and reported strong associations.

Taken together, this study is the first single cohort study to perform a comprehensive analysis of modulatory role of 13 most frequently studied SNPs with clinical outcome of MTC patients. We have extended our analysis on both the hereditary and sporadic MTC cohort and reported no significant association of these SNPs with clinical behavior of MTC. There is a need for additional genetic association studies on large cohort of MTC cases with clinic-pathological details and long-term follow-up data in order to draw consistent and definitive conclusions.

\section{Acknowledgements}

We thank the Indian Council of Medical Research for funding the project and Department of Science and Technology, Government of India for providing fellowship to Ms Vasudha Mishra. We acknowledge the cooperation of members of the Head and Neck Disease Management Group, Tata Memorial Hospital (TMH) for referring the patients and Mr. Ravindra Reddy and other genetic counselors at Cancer Genetics Clinic, TMH for providing counseling to the patients. We are thankful to all the patients and their family members for their participation in the study.

\section{Disclosure}

The authors declare no conflict of interest. 


\section{Data Availability Statement}

The data that support the findings of this study are available from the corresponding author upon reasonable request.

\section{References}

Agrawal N, Jiao Y, Sausen M, et al (2013). Exomic sequencing of medullary thyroid cancer reveals dominant and mutually exclusive oncogenic mutations in RET and RAS. $J$ Clin Endocrinol Metab, 98, 364-9.

Barbieri RB, Bufalo NE, Secolin R, et al (2012). Evidence that polymorphisms in detoxification genes modulate the susceptibility for sporadic medullary thyroid carcinoma. Eur J Endocrinol, 166, 241-5.

Barbieri RB, Bufalo NE, Cunha LL, et al (2013). Genes of detoxification are important modulators of hereditary medullary thyroid carcinoma risk. Clin Endocrinol, 79, 288-93.

Barbieri RB, Bufalo NE, Secolin R, et al (2014). Polymorphisms of cell cycle control genes influence the development of sporadic medullary thyroid carcinoma. Eur J Endocrinol, 171, 761-7.

Cardot-Bauters C, Leteurtre E, Leclerc L, et al (2008). Does the RET variant G691S influence the features of sporadic medullary thyroid carcinoma?. Clin Endocrinol, 69, 506-10.

De Groot JWB, Links TP, Plukker JTM, et al (2006). RET as a diagnostic and therapeutic target in sporadic and hereditary endocrine tumors. Endocr Rev, 27, 535-60.

Donis-Keller H, Dou S, Chi D, et al (1993). Mutations in the RET proto-oncogene are associated with MEN2A and FMTC. Hum Mol Genet, 2, 851-6.

Eng C, Clayton D, Schuffenecker I, Al E (1996). The relationship between specific ret proto-oncogene mutations and disease phenotype in multiple endocrine neoplasia type 2: International ret mutation consortium analysis. JAMA, 276, 1575-9.

Eng C, Mulligan LM, Healey CS, et al (1996). Heterogeneous mutation of the RET proto-oncogene in subpopulations of medullary thyroid carcinoma. Cancer Res, 56, 2167-70.

Figlioli G, Landi S, Romei C, Elisei R, Gemignani F (2013). Medullary thyroid carcinoma (MTC) and RET proto-oncogene: Mutation spectrum in the familial cases and a meta-analysis of studies on the sporadic form. Mutat Res, 752, 36-44.

Fugazzola L, Muzza M, Mian C, et al (2008). RET genotypes in sporadic medullary thyroid cancer: studies in a large Italian series. Clin Endocrinol, 69, 418-25.

Jensen MM, Jorgensen JT, Binderup T, Kjaer A (2008). Tumor volume in subcutaneous mouse xenografts measured by microCT is more accurate and reproducible than determined by $18 \mathrm{~F}-\mathrm{FDG}-\mathrm{microPET}$ or external caliper. $\mathrm{BMC} \mathrm{Med}$ Imag, 8.

Kloos RT, Eng C, Evans DB, et al (2009). Medullary thyroid cancer: Management Guidelines of the American Thyroid Association. Thyroid, 19, 565-612.

Lesueur F, Cebrian A, Robledo M, et al (2006). Polymorphisms in RET and its coreceptors and ligands as genetic modifiers of multiple endocrine neoplasia type 2A. Cancer Res, 66, 1177-80.

Machens A, Frank-Raue K, Lorenz K, et al (2012). Clinical relevance of RET variants G691S, L769L, S836S and S904S to sporadic medullary thyroid cancer. Clin Endocrinol, 76, 691-7.

Marsh DJ, Learoyd DL, Andrew SD, et al (1996). Somatic mutations in the RET proto-oncogene in sporadic medullary thyroid carcinoma. Clin Endocrinol, 44, 249-57.
Pasquali D, Circelli L, Faggiano A, et al (2011). CDKN1B V109G polymorphism a new prognostic factor in sporadic medullary thyroid carcinoma. Eur J Endocrinol, 164, 397-404.

Robledo M, Gil L, Pollan M, et al (2003). Polymorphisms G691S/S904S of RET as genetic modifiers of MEN 2A. Cancer Res, 63, 1814-7.

Siqueira DR, Romitti M, da Rocha AP, et al (2010). The RET polymorphic allele S836S is associated with early metastatic disease in patients with hereditary or sporadic medullary thyroid carcinoma. Endocr Relat Canc, 17, 953-63.

Sromek M, Czetwertynska M, Skasko E, et al (2010). The frequency of selected polymorphic variants of the RET gene in patients with medullary thyroid carcinoma and in the general population of central Poland. Endocr Pathol, 21, 178-85.

Tamanaha R, Camacho CP, Pereira AC, et al (2009). Evaluation of RET polymorphisms in a six-generation family with G533C RET mutation: specific RET variants may modulate age at onset and clinical presentation. Clin Endocrinol, 71, 56-64.

Wells SA, Asa SL, Dralle H, et al (2015). Revised American Thyroid Association Guidelines for the Management of Medullary Thyroid Carcinoma. Thyroid, 25, 567-610.

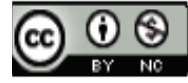

This work is licensed under a Creative Commons AttributionNon Commercial 4.0 International License. 